\title{
Towards the MSSM from F-theory
}

\author{
Kang-Sin Choi, Tatsuo Kobayashi \\ Department of Physics, Kyoto University, Kyoto 606-8502, Japan
}

\begin{abstract}
We study the MSSM in F-theory. Its group is the commutant to a structure group $S U(5) \times U(1)_{Y}$ of a gauge bundle in $E_{8}$. The spectrum contains three generations of quarks and leptons plus vectorlike electroweak and colored Higgses. The minimal MSSM Yukawa couplings with matter parity is obtained at the renormalizable level.
\end{abstract}

\section{Introduction}

The purpose of this letter is to describe the Minimal Supersymmetric Standard Model (MSSM) in F-theory. String theory provides self-consistent ultraviolet completion of the gauge theory. For example, the constraint of anomaly freedom in the low energy theory originates from the finiteness of string one-loop amplitude.

F-theory is defined by identifying $S$-duality of IIB the'ory with the symmetry of a torus, lifting the gauge symmetry to geometry [1]. To have four dimensional theory 'with $\mathcal{N}=1$ supersymmetry, we compactify F-theory on Calabi-Yau fourfold that is elliptic fibered. Gauge bosons are localized on a complex surface $S$, along which the fiber is singular. The structure of the singularities has correspondence to that of the corresponding group, so that the symmetry breaking and enhancement are described by geometric transition [2]. Analogous to the bifundamental representations at D-brane intersections, matter fermions 'come from the 'off-diagonal' components under branching of the gaugino of $E_{8}[3]$, localized along matter curves [4].

So far there have been constructions of Grand Unified Theories (GUTs) mainly based on the simple group $S U(5)$, and further attention has been paid on the flavor sector [5, 6, 7]. Compared to field theoretic GUT, presumably its best merit is that we do not need adjoint Higgses for breaking down to SM. Instead, we can turn on a flux in the hypercharge $U(1)_{Y}$ direction, evading complicated GUT vacuum configuration. This flux does not break hypercharge interaction, if a certain topological condition is satisfied [6]. However, further breaking down to SM can potentially lead to chiral multiplet containing $X$-boson, the off-diagonal component of the adjoint of $S U(5)$, by the above mechanism localizing light matters.

Another obvious approach that we take here is to start with the group $S U(3) \times S U(2) \times U(1)_{Y}$. The SM group lies

Email addresses: kschoi@gauge.scphys.kyoto-u.ac.jp (Kang-Sin Choi), kobayash@gauge.scphys.kyoto-u.ac.jp (Tatsuo Kobayashi) along the series of exceptional groups of $E_{n}$-type, and we take $E_{3} \times U(1)_{Y}$ group inside $E_{8}$, shown in Fig. 1] guaranteeing the correct field contents and quantum numbers. Such $E_{n}$-series is naturally predicted by F-theory and heterotic string. For this we need a description on semisimple groups [8, 9]. If $U(1)_{Y}$ is constructed via geometry and there is no flux along this part, embeddability to $S U(5)$ GUT singularity guarantees anomaly free spectrum and we do not worry about its breaking by Green-Schwarz mechanism.

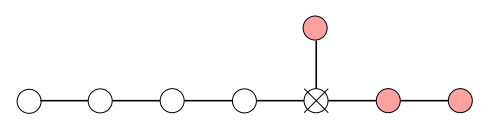

Figure 1: The Standard Model group (filled) is obtained as the commutant to $S U(5)_{\perp} \times U(1)_{Y}$ background in $E_{8}$.

\section{The Standard Model surface}

A gauge group is described by a singular fiber sharing the same name, which is read off from Tate's table [10]. We claim that the singularity describing the SM group is

$$
\begin{aligned}
y^{2} & =x^{3}+\left(b_{5}+b_{4} a_{1}\right) x y+\left(b_{3}+b_{2} a_{1}\right)\left(a_{1} b_{5}+z\right) z y \\
& +\left(b_{4}+b_{3} a_{1}\right) x^{2} z+\left(b_{2}-b_{0} a_{1}^{2}\right)\left(a_{1} b_{5}+z\right) x z^{2} \\
& +b_{0}\left(a_{1} b_{5}+z\right)^{2} z^{3} .
\end{aligned}
$$

For the total space to be Calabi-Yau, the equation should satisfy topological conditions: $z, a_{1}, b_{n}$ are respectively sections of $\mathcal{O}(S), \mathcal{O}\left(K_{B}+S\right), \mathcal{O}\left((n-6) K_{B}+(n-5) S\right)$ in the base $B$ of elliptic fibration, and $K_{B}$ is its canonical bundle. The surface $S$ is located at $z=0$, since the vanishing discriminant of (1)

$$
\Delta=\left(b_{5}+a_{1} b_{4}\right)^{3} a_{1}^{2} b_{5}^{2} P_{d_{\circ}^{c}} P_{u_{\circ}^{c}} z^{3}+a_{1} b_{5} Q z^{4}+O\left(z^{5}\right)
$$

signals a singular fiber. We need globally defined sections in $B$, since the simple group components would be laid away from $S$. 
Eq. (11) is a deformation of $E_{8}$ singularity. Surveying the degrees of the coefficients in (11), Tate's table shows it is generically an $S U(3)$ singularity. However the parameters are specially tuned, so the actual symmetry is larger [8]. Vanishing of each coefficient factor triggers gauge symmetry enhancement, implying matter localization [2]. We see shortly that the parameters $a_{1} \equiv P_{X}, b_{5} \equiv P_{q_{\circ}}, P_{d_{\circ}^{c}}$ and $P_{u_{\circ}^{c}}$ are respectively related to $X$-boson and subscripted quarks, which are all the charged fields under $S U(3)$. We can see, neither $Q$ nor $O\left(z^{5}\right)$ is proportional to $a_{1}$ or $b_{5}$, hence $P_{d_{\circ}^{c}} P_{u_{\circ}^{c}}$ and $a_{1} b_{5} \rightarrow 0$ respectively enhance the symmetry to $S U(4)$ and $S U(5)$.

To see it contains also $S U(2)$, we change the variable $z^{\prime}=z+a_{1} b_{5}$, in which (2) becomes

$$
\begin{aligned}
\Delta= & \left(\left(b_{5}-a_{1} b_{4}\right)^{2}-4 a_{5}^{2} b_{3} b_{5}\right)^{2} a_{1}^{3} b_{5}^{3} P_{l_{\circ}} z^{2} \\
& +a_{1}^{2} b_{5}^{2} P^{\prime} z^{\prime 3}+a_{1} b_{5} Q^{\prime} z^{\prime 4}+O\left(z^{5}\right) .
\end{aligned}
$$

Here $P_{l}$ is related to the lepton doublet. Since neither of $P^{\prime}, Q^{\prime}$ nor $O\left(z^{\prime 5}\right)$ is proportional to $a_{1}$ or $b_{5}$, vanishing $P_{l^{\circ}}$ and $a_{1} b_{5}$ respectively enhances the gauge symmetry respectively to $S U(3)$ and $S U(5)$. The position $z^{\prime}=0$ is off from the position of $S$, as a 'back-reaction' under the symmetry breaking from $S U(5)$, but it is still linearly equivalent to $S$. We can see, to make the embracing $S U(5)$ truly traceless, we need also a backreaction $z \rightarrow z-2 a_{1} b_{5} / 5$ to make the center of mass of the total brane lie on $S$.

The factorization structure of $P_{u_{\circ}^{c}} P_{d_{\circ}^{c}}$ and $P_{X} P_{q_{\circ}}=a_{1} b_{5}$ hints the existence of an additional $U(1)_{Y}$, otherwise we have only single factors for colored singlet and colored doublet, respectively. However to guarantee the existence of such $U(1)_{Y}$ we should check the global factorization structure of (11) 11]. Above all, the most general deformation is by $a_{1} \rightarrow 0$, implying the embedding of our symmetry to a general $S U(5)$ GUT singularity in the literature [3]. Being its deformation, the factorization structure of $S U(3) \times S U(2) \times U(1)_{Y}$ is stably preserved against higher order perturbation in $O\left(z^{6}\right)$. Lying along the $E_{n}$ unification series $E_{3} \times U(1)_{Y} \rightarrow E_{4} \rightarrow E_{8}$, this $\mathrm{SM}$ group is the only possibility.

\section{Matter contents}

The broken symmetry is $S U(5)_{\perp} \times U(1)_{Y}$ 'structure group' whose commutant in $E_{8}$ is the Standard Model group [12]. The spectral cover geometrically describes it (or the dual to gauge bundle) satisfying supersymmetry conditions [13]. Roughly, it generalizes the notion of branes whose symmetry broken by recombination, so sometimes called by flavor brane stack, intersecting $S$ along the matter curves. It is described by factorized spectral cover $C=C_{X} \cup C_{5}$ :

$$
\left(a_{0} s+a_{1}\right)\left(b_{0} s^{5}-a_{0}^{-1} a_{1} b_{0} s^{4}+b_{2} s^{3}+b_{3} s^{2}+b_{4} s+b_{5}\right)=0,
$$

where $s$ transforms as $-c_{1} \equiv K_{S}$, the canonical bundle of $S$. We also define $-t$ as the normal bundle to $S$ in $B$. (4) is embedded in a compact threefold 1 , whose projection to $S$ we denote $\pi$. The parameters are the ones used in (11) projected on $S$ : Using adjunction formula $b_{m}$ are sections of $(6-m) c_{1} \equiv \eta-m c_{1}$ and the combination $a_{1} b_{0} / a_{0}$ is a section of $5 c_{1}-t$, with which the full equation for $C$ has no $s^{5}$ term showing the embeddability to a $S U(6)_{\perp}$ structure group 2. Using this embedding structure, one can be convinced that the parameters in (1) are the only possible combinations. We can recover the conventional $S U(5)$ GUT by $a_{1} \rightarrow 0$ in (2), making its structure group traceless, reducing the above $C_{5}$ exactly to the standard one used in $S U(5)$ GUT. The fields charged under two groups satisfy the Green-Schwarz relations [21, 8], fixing $a_{0}=1$ to be trivial section.

We get the matter spectrum from the decomposition of the adjoint 248 of $E_{8}$

$$
\begin{aligned}
& (\mathbf{8}, \mathbf{1}, \mathbf{1})+(\mathbf{1}, \mathbf{3}, \mathbf{1})+(\mathbf{1}, \mathbf{1}, \mathbf{1})+(\mathbf{1}, \mathbf{1}, \mathbf{2 4}) \\
& +X(\mathbf{3}, \mathbf{2}, \mathbf{1})_{-5 / 6}+q_{\circ}(\mathbf{3}, \mathbf{2}, \mathbf{5})_{1 / 6}+d_{\circ}^{c}(\overline{\mathbf{3}}, \mathbf{1}, \mathbf{1 0})_{1 / 3} \\
& +u_{\circ}^{c}(\overline{\mathbf{3}}, \mathbf{1}, \mathbf{5})_{-2 / 3}+l_{\circ}(\mathbf{1}, \mathbf{2}, \mathbf{1 0})_{-1 / 2}+e_{\circ}^{c}(\mathbf{1}, \mathbf{1}, \overline{\mathbf{5}})_{-1},
\end{aligned}
$$

up to Hermitian conjugates. Matter fields are obtained as 'off-diagonal' components of the branching [2].

They are further identified by local gauge symmetry enhancement directions. For this, we parameterize their localizing curves using parameters $t_{1}, t_{2}, \ldots t_{5}$ having one-toone correspondence with the five weights of $\mathbf{5}$ of $S U(5)_{\perp}$ and $t_{6}$ with $U(1)_{Y}$. For example, the $X$-boson appears from a local symmetry enhancement to $S U(5)$, controlled by $t_{6} \rightarrow 0$, agreeing with the above. However the physical parameters are only the coefficients in the spectral cover (41): $b_{n} / b_{0}$ are the elementary symmetric polynomials of degree $k$, of $t_{1}, t_{2}, t_{3}, t_{4}, t_{5}$, and $a_{1} \equiv P_{X} \sim t_{6}$ [5, 3]. $P_{q_{\circ}}$ is localized along the curve $b_{5} / b_{0} \sim t_{1} t_{2} t_{3} t_{4} t_{5}=0$ and $P_{u_{\circ}^{c}} \sim$ $\prod_{i=1}^{5}\left(t_{i}+t_{6}\right), P_{d_{\circ}^{c}} \sim \prod_{i<j}^{5}\left(t_{i}+t_{j}\right), P_{l_{\circ}} \sim \prod_{i<j}^{5}\left(t_{i}+t_{j}+t_{6}\right)$. The counting of $\mathbf{1 0}$ and $\overline{\mathbf{1 0}}$ agrees thanks to the relation

$$
t_{i}+t_{j}+t_{6}=-t_{k}-t_{l}-t_{m}, \quad \epsilon_{i j k l m} \neq 0 .
$$

It is easy to see that the parameters $P_{m_{\circ}}, m=$ $X, q, u^{c}, d^{c}, l$, here calculated from group theory, agree with those in (2) and (3). Again $t_{6} \rightarrow 0$ reduces the matter curves to those of $S U(5)$, so the $S U(5)$ GUT structure is preserved.

In the perturbative picture, parallel D-branes do not intersect. Here, the base of elliptic fibration has a similar structure generalizing Hirzeburch surface, where the zero section of the fiber has nonzero 'self-intersection'. Thus $S U(3)$ and $S U(2)$ components intersect yielding chiral fermions $X$ and $q$. In the sense of regarding the intersecting branes as connected cycles [15], this is not so different.

\footnotetext{
${ }^{1}$ The compact (non-Calabi-Yau) threefold, a projectivized fiber $\pi: \mathbb{P}\left(\mathcal{O}_{S} \oplus K_{S}\right) \rightarrow S$, with the trivial bundle $\mathcal{O}_{S}$ and the canonical bundle $K_{S}$.

${ }^{2}$ Since the trace part of $S U(5)_{\perp}$ is the $U(1)_{Y}$ degree of freedom, it is sometimes called $S[U(5) \times U(1)]$.
} 
By dimensional reduction, we obtain Yukawa coupling from covariant derivatives of gaugino of an enhanced group, as usual [5]. So the gauge invariance guarantees the presence of Yukawa couplings. They are

$$
\begin{aligned}
l h_{d} e^{c} & :\left(t_{i}+t_{j}+t_{6}\right)+\left(t_{k}+t_{l}+t_{6}\right)+\left(t_{m}-t_{6}\right)=0, \\
q h_{u} u^{c}: & :\left(t_{i}\right)+\left(-t_{i}-t_{j}-t_{6}\right)+\left(t_{j}+t_{6}\right)=0, \\
q h_{d} d^{c}: & : \begin{array}{c}
\left(t_{m}\right)+\left(t_{k}+t_{l}+t_{6}\right)+\left(t_{i}+t_{j}\right)=0, \\
\left(t_{m}\right)+\left(-t_{m}-t_{i}-t_{j}\right)+\left(t_{i}+t_{j}\right)=0,
\end{array}
\end{aligned}
$$

where all the indices run from 1 to 5 , and all different.

Due to the relation (5), LHS's of (6) vanish, and in each relation, we have two or more ways of writing the same coupling. For example in the last line, the first relation reduces the same relation to that of $S U(5) \mathrm{GUT}$, in the limit $t_{6} \rightarrow 0$. The second relates the matter curves for all $q, h_{d}, d^{c}, h_{u}$ and $u^{c}$. We may track this from the leftright symmetric models and their extension (Pati-Salam and trinification) which relates up and down sectors by $S U(2)_{R}$. We can switch between up-type and down-type fermions, like doing between Georgi-Glashow $S U(5)$ and flipped $S U(5)$.

\section{Matter curves and monodromy}

For a general spectral cover, we have monodromy condition for connecting different matter curves [16]. In our case, the $S U(5)_{\perp}$ has $S_{5}$ monodromy as reflected in the symmetric polynomial relations of the coefficients: The connected ones form an orbit and are treated as the identical surface. It leads to just one kind of lepton doublet, without distinguishing $l, h_{d}$ or $h_{u}^{c}$.

To remedy this, we further mod out the others by $\mathbb{Z}_{4}$ monodromy generated by the cyclic permutation of $\left(t_{1}, t_{2}, t_{3}, t_{4}\right)$. It follows that, for instance, $q$ is distinguished by different orbits $\left\{t_{1}, t_{2}, t_{3}, t_{4}\right\}$ and $\left\{t_{5}\right\}$, whereas $X$ is still $\left\{t_{6}\right\}$. There are distinct candidates for the matters. For example lepton or Higgs belongs to one of the following $\mathbb{Z}_{4}$ orbit

$l, h_{d}, h_{u}^{c}:\left\{t_{i}+t_{i+1}+t_{6}\right\},\left\{t_{1}+t_{3}+t_{6}, t_{2}+t_{4}+t_{6}\right\},\left\{t_{i}+t_{5}+t_{6}\right\}$,

with $i=1, \ldots, 4$. To have lepton Yukawa coupling (6), $h_{d}$ and $l$ must not share $t_{5}$, therefore essentially we have two allowed cases for choosing $S U(2)$ doublets. We choose the fields as in Table 1. We named the colored exotics as colored Higgses $h_{c 1}$ and $h_{c 2}$. Note also that, by $S U(5)$ unification structure the fields belonging to a single multiplet has homologous curves.

We identify two Abelian symmetries generated by

$$
\begin{gathered}
U(1)_{Y}: \operatorname{diag}(1,1,1,1,1,-5), \\
U(1)_{M}: \operatorname{diag}(1,1,1,1,-4,0),
\end{gathered}
$$

in the basis $\left\{t_{1}, t_{2}, t_{3}, t_{4}, t_{5}, t_{6}\right\}$. The first is $U(1)_{Y}$ and the second is the famous ' $B-L$ ' symmetry, commutant

\begin{tabular}{cccc}
\hline matter & matter curve & cycle on $S$ & $M$ \\
\hline$X$ & $t_{6} \rightarrow 0$ & $-c_{1}$ & 0 \\
$q$ & $\prod t_{i} \rightarrow 0$ & $\eta-4 c_{1}-x$ & 1 \\
$q^{\prime}$ & $t_{5} \rightarrow 0$ & $-c_{1}+x$ & -4 \\
$d^{c}$ & $\prod\left(t_{i}+t_{5}\right) \rightarrow 0$ & $\eta-4 c_{1}+2 x$ & -3 \\
$h_{c 1}^{c}$ & $\prod\left(t_{i}+t_{i+2}\right) \rightarrow 0$ & $\eta-2 c_{1}-x$ & 2 \\
$h_{c 2}$ & $\prod\left(t_{i}+t_{i+1}\right) \rightarrow 0$ & $\eta-4 c_{1}-x$ & 2 \\
$u^{c}$ & $\prod\left(t_{i}+t_{6}\right) \rightarrow 0$ & $\eta-4 c_{1}-x$ & 1 \\
$u^{\prime}$ & $t_{5}+t_{6} \rightarrow 0$ & $-c_{1}+x$ & -4 \\
$h_{u}^{c}$ & $\prod\left(t_{i}+t_{i+1}+t_{6}\right) \rightarrow 0$ & $\eta-4 c_{1}-x$ & 2 \\
$h_{d}$ & $\prod\left(t_{i}+t_{i+2}+t_{6}\right) \rightarrow 0$ & $\eta-2 c_{1}-x$ & 2 \\
$l$ & $\prod\left(t_{i}+t_{5}+t_{6}\right) \rightarrow 0$ & $\eta-4 c_{1}+2 x$ & -3 \\
$e^{c}$ & $\prod\left(t_{i}-t_{6}\right) \rightarrow 0$ & $\eta-4 c_{1}-x$ & 1 \\
$e^{\prime}$ & $t_{5}-t_{6} \rightarrow 0$ & $-c_{1}+x$ & -4 \\
\hline \multicolumn{5}{c}{}
\end{tabular}

Table 1: Matter curves with $\mathbb{Z}_{4}$ monodromy. All the indices take value in $\mathbb{Z}_{4}=\{1,2,3,4\}$ and are different. The primed ones, charged exotics, have always odd $M+2 s$ charges.

to $S U(5)$ inside $S O(10)$, providing continuous version of matter parity. Since both $h_{u}$ and $h_{d}^{c}$ have even $U(1)_{M}$ charges, we can forbid lepton or baryon number violating operators

$$
l h_{u}, l l e^{c}, l q d^{c}, u^{c} d^{c} d^{c}
$$

We may also have operators

$$
X h_{u} d^{c}, q^{\prime} h_{d}^{c} h_{c 1}^{c}, q^{\prime} h_{u} h_{c 2}
$$

It turns out that neither $X$ nor $q^{\prime}$ exists in four dimensions, for later choice of the flux (see (13) below), so there will be no problem.

The resulting spectral cover is further factorized $C_{5} \rightarrow$ $C_{q^{\prime}} \cup C_{q}$, thus (4) becomes

$$
\left(s+a_{1}\right)\left(d_{0} s+d_{1}\right)\left(e_{0} s^{4}+e_{1} s^{3}+\left(e_{2}^{\prime}+e_{2}^{\prime \prime}\right) s^{2}+e_{3} s+e_{4}\right)=0
$$

with 'traceless condition' $a_{1} d_{0} e_{0}+d_{1} e_{0}+d_{0} e_{1}=0$. The new covers are for $t_{5} \sim d_{1} / d_{0}$, and $e_{i}$ will be again related to elementary symmetry polynomials of degree $i$ out of $t_{1}, t_{2}, t_{3}, t_{4}$. Only $e_{2}^{\prime} / e_{0} \sim\left(t_{1}+t_{3}\right)\left(t_{2}+t_{4}\right)$ and $e_{2}^{\prime \prime} / e_{0} \sim$ $t_{1} t_{3}+t_{2} t_{4}$ are nontrivially $\mathbb{Z}_{4}$ closed. We have additional degree of freedom to choose $d_{0}$, a section $x \in H_{2}(S, \mathbb{Z})$. It follows $e_{k}$ are sections $e_{k} \sim \eta-k c_{1}-x$.

As discussed around (6), there are fields related by nonvanishing Yukawa couplings. They originate from the same spectral cover. Since the defining conditions $t_{i}=0$ are stronger than $t_{i}+t_{j}=0$, we can derive all associated matter curves from the 'fundamental' spectral covers $t_{i}=0$ for $C_{X}, C_{q^{\prime}}, C_{q}[3,17]$. For example the matter curve for $d^{c}$ is the common intersection between $C_{q}\left(t_{i}\right)$ and $C_{q^{\prime}}\left(-t_{i}\right)$, extracting the redundant component.

From the symmetry enhancement directions, as shown in Table 1, we can find the following classes of the matter 
curves:

$$
\begin{aligned}
& X: C_{X} \cap \sigma=-c_{1} \cap \sigma \\
& q, u^{c}, e^{c}: C_{q} \cap \sigma=C_{q} \cap C_{X}=\left(\eta-4 c_{1}-x\right) \cap \sigma \\
& q^{\prime}, u^{\prime}, e^{\prime}: C_{q^{\prime}} \cap \sigma=C_{q^{\prime}} \cap C_{X}=\left(-c_{1}+x\right) \cap \sigma \\
& d^{c}, l:\left(C_{q}-\left(\sigma+c_{1}\right)\right) \cap C_{q^{\prime}} \\
&=\sigma \cap\left(\eta-4 c_{1}+2 x\right)+\left(\eta-c_{1}-x\right) \cap x \\
& h_{c 1}, h_{d}: C_{q} \cap\left(2 \sigma+c_{1}\right) \\
&=2 \sigma \cap\left(\eta-2 c_{1}-x\right)+(\eta-x) \cap c_{1} \\
& h_{c 2}^{c}, h_{u}^{c}:\left(C_{q}-2 \sigma\right) \cap\left(C_{q}-4\left(\sigma+c_{1}\right)\right) \\
&=2 \sigma \cap\left(\eta-4 c_{1}-x\right)+(\eta-x) \cap\left(\eta-4 c_{1}-x\right)
\end{aligned}
$$

where we omitted pullback. The associated matter curves have $\sigma$-independent components that lie outside $S$ but on the spectral cover. Since $a_{0}$ is trivial section, thus $C_{X} \sim \sigma$ and the matter curve with and without $t_{6}$ are homologous, for $q, u^{c}, e^{c}$ for example. Again this exhibits the unification relations to $S U(5)$ GUT. More detailed calculation is to be found elsewhere [18].

\section{Flux and spectrum}

To obtain four dimensional chiral spectrum, we should turn on a magnetic flux $\gamma$ on the spectral cover $\gamma \in$ $H^{2}(C, \mathbb{Z})[13,14$. With above factorization we turn on the universal flux only on $C_{q}$ 3

$$
\gamma_{q}=C_{q} \cap\left(4 \sigma-\pi^{*}\left(\eta-4 c_{1}\right)\right), \quad \gamma_{q^{\prime}}=\gamma_{X}=0 .
$$

We can show it has integral cohomology and traceless $p_{C *} \gamma=0$ inside $S U(5)_{\perp} \times U(1)_{Y}$, where $p_{C}: C \rightarrow S$. It follows that $q^{\prime}, X$ and the associated matters are all neutral to have zero multiplicity

$$
n_{X}=n_{q^{\prime}}=n_{u^{\prime}}=n_{e^{\prime}}=0 .
$$

We calculate the net number of fields, or the differences $n_{f}$ between the numbers of fermions $f$ and antifermions $f^{c}$ by Riemann-Roch-Hirzebruch index theorem. It assumes the same form for associated matter curves, namely the product of $4 \sigma-p_{q}^{*}\left(\eta-4 c_{1}\right)$ with the curves in (11), letting $p_{q}$ the projection $C_{q} \rightarrow S[20,19]$. For example, we obtain

$$
\begin{aligned}
n_{q}= & \left.\sigma \cap\left(\eta-4 c_{1}-x\right) \cap\left(4 \sigma-p_{q}^{*}\left(\eta-4 c_{1}\right)\right)\right|_{S} \\
= & -\eta \cdot\left(\eta-4 c_{1}-x\right) \\
n_{d^{c}}= & \left(\sigma \cap\left(\eta-4 c_{1}+2 x\right)+\left(\eta-c_{1}-x\right) \cap x\right) \\
& \left.\cap\left(4 \sigma-p_{q}^{*}\left(\eta-4 c_{1}\right)\right)\right|_{S} \\
= & -\eta \cdot\left(\eta-4 c_{1}+2 x\right)+4\left(\eta-c_{1}-x\right) \cdot x,
\end{aligned}
$$

where the dot product - means the intersection between the divisors on $S$ and we used the Poincaré dual flux. In

\footnotetext{
${ }^{3}$ There is no ramification on $C_{q^{\prime}}$ and $C_{X}$ parts, so we can turn off fluxes on them, and the even (fourfold) cover of $C_{q}$ allows that integral $\gamma_{q}$ corresponds to integral spectral line bundle [19].
}

fact, Kodaira vanishing theorem states that either chiral or antichiral fermion has exclusively nonzero in most cases 5 , 6]. The anomaly cancellation condition for $S U(3), 2 n_{q}-$ $n_{d^{c}}-n_{u^{c}}=0$, requires also $x=0$ or $4 x=5 \eta-c_{1}$. We choose $x=0$ to have the spectrum

$$
\begin{aligned}
& n_{q}=n_{d^{c}}=n_{u^{c}}=n_{l}=n_{e^{c}}=-\eta \cdot\left(\eta-4 c_{1}\right), \\
& n_{h_{d}}=n_{h_{c 1}}=n_{h_{c 2}}=n_{h_{u}}=-2 \eta \cdot\left(\eta-4 c_{1}\right) .
\end{aligned}
$$

Given the base $B$ of elliptic fibration, $c_{1},-t$ are determined purely by the property of $S$. Choosing $S$ such that $-\eta$. $\left(\eta-4 c_{1}\right)=3$, we get three generations of SM fermions, plus six pairs of doublet Higgses and six pairs of colored Higgses. Since we make use of the section $-c_{1}$ for $U(1)_{Y}$ we need $h^{0}\left(S, K_{S}\right)>0$. However it might imply the SM adjoint Higgses unless $S$ is torsion-free.

We obtained the SM gauge group and three generations of quarks and leptons without resorting to an intermediate unification. For the vectorlike electroweak and colored Higgses, we have the standard doublet-triplet splitting problem, also tightly related to the $\mu$-problem. We can further elaborate the model employing different factorization and/or flux. Already this model has desirable symmetries to shed light on a dynamical resolution. Because of gauge invariance (6) and the vanishing theorem, bare mass terms of F-theory scale, close to the Planck scale, are forbidden. However below some intermediate scale $M_{I}$ where the global symmetries and $U(1)_{M}$ are broken, mass terms would be dynamically generated

$$
W=W_{\mathrm{MSSM}}(\mu=0)+m_{c} h_{c 1} h_{c 2}+m_{h} h_{u} h_{d} .
$$

We expect some 'standard solution' would generate the mass matrix $m_{c}$ and $m_{h}$ at the scale $M_{I}$. We again emphasize the corresponding fields are distinguished by $U(1)_{M}$ charges in Table 1. We expect it would be broken down to $\mathbb{Z}_{2}$ symmetry, becoming matter parity 22 . Then still the terms (8) are forbidden in the low energy. Also this structure can be related to dynamical supersymmetry breaking [23].

The authors acknowledge the support by the Grant-inAid for Scientific Research No. 20.08326 and 20540266 from the Ministry of Education, Culture, Sports, Science and Technology of Japan (MEXT). T. K. is also supported in part by the Grant-in-Aid for the Global COE Program "The Next Generation of Physics, Spun from Universality and Emergence" from MEXT.

\section{References}

[1] C. Vafa, Nucl. Phys. B 469, 403 (1996).

[2] S. H. Katz and C. Vafa, Nucl. Phys. B 497, 146 (1997).

[3] R. Donagi and M. Wijnholt, arXiv:0904.1218 [hep-th].

[4] D. R. Morrison and C. Vafa, Nucl. Phys. B 476, 437 (1996); Nucl. Phys. B 473, 74 (1996).

[5] C. Beasley, J. J. Heckman and C. Vafa, JHEP 0901, 058 (2009); JHEP 0901, 059 (2009).

[6] R. Donagi and M. Wijnholt, arXiv:0808.2223 [hep-th].

[7] See J. J. Heckman, arXiv:1001.0577 [hep-th] and references therein. 
[8] K. S. Choi, JHEP 1002, 004 (2010).

[9] S. Katz and D. R. Morrison, J. Algebraic Geom. 1 (1992), 449.

[10] M. Bershadsky et. al. Nucl. Phys. B 481, 215 (1996).

[11] H. Hayashi, T. Kawano, Y. Tsuchiya and T. Watari, arXiv:1004.3870 [hep-th]; T. W. Grimm and T. Weigand, arXiv:1006.0226 [hep-th].

[12] In the heterotic language. See, e.g. R. Blumenhagen, S. Moster and T. Weigand, Nucl. Phys. B 751, 186 (2006).

[13] R. Friedman, J. Morgan and E. Witten, Commun. Math. Phys. 187, 679 (1997).

[14] See also H. Abe, K. S. Choi, T. Kobayashi and H. Ohki, Phys. Rev. D 80, 126006 (2009); K. S. Choi et. al., Eur. Phys. J. C 67, 273 (2010).

[15] K. S. Choi, Phys. Rev. D 74, 066002 (2006); Int. J. Mod. Phys. A 22, 3169 (2007); K. S. Choi and J. E. Kim, JHEP 0511 (2005) 043.

[16] H. Hayashi, T. Kawano, R. Tatar and T. Watari, Nucl. Phys. B 823, 47 (2009).

[17] J. Marsano, N. Saulina and S. Schafer-Nameki, JHEP 0908, 046 (2009).

[18] K. S. Choi, arXiv:1007.3843 [hep-th].

[19] R. Blumenhagen, T. W. Grimm, B. Jurke and T. Weigand, Nucl. Phys. B 829, 325 (2010).

[20] H. Hayashi, R. Tatar, Y. Toda, T. Watari and M. Yamazaki, Nucl. Phys. B 806, 224 (2009).

21] V. Sadov, Phys. Lett. B 388, 45 (1996).

[22] See also J. E. Kim, J. H. Kim and B. Kyae, JHEP 0706, 034 (2007); O. Lebedev et. al. Phys. Rev. D 77 (2008) 046013.

[23] J. Marsano, N. Saulina and S. Schafer-Nameki, JHEP 0908 (2009) 030. 